\title{
What Determines China's Stock Prices? A CCAPM Horse Race
}

\author{
Jinyu Liu' ${ }^{1}$ and Siqun Yang ${ }^{2}$
}

\begin{abstract}
This paper studies China's stock prices in the framework of consumption-based capital asset pricing models (CCAPM). Using China's quarterly stock market data from 1991 to 2019, we estimate and compare four versions of CCAPM: the classical CCAPM, CCAPM with housing service consumption, with habit formation, and with both. We find habit formation affects stock returns only if housing service consumption is considered. Further, although every model is consistent with data to certain extent, the models with habit formation perform substantially better. In particular, the model with both habit formation and housing service consumption fits the data best and has the largest explanatory power on risk premium. The findings imply that habit formation is an important determinant of China's stock prices, and its impact is mostly via the channel of housing service consumption.
\end{abstract}

JEL classification numbers: E20, G10

Keywords: Asset pricing, consumption-based capital asset pricing model, risk premium, habit formation.

\footnotetext{
1 Department of Economics, School of Economics and Management, Tsinghua University, Beijing, China..

2 Department of Economics, School of Economics and Management, Tsinghua University, Beijing, China.
} 


\section{Introduction}

Understanding the mechanism of asset price determination is a central topic in finance and economics. With distinct cultural and economic backgrounds from other major economies, it is important to understand the asset markets in China.

Among various approaches to this goal, consumption-based capital asset pricing models (CCAPM, see Lucas, 1978, Mehra and Prescott, 1985, Campbell, 1986 etc.) are popular tools as they have a well-established micro-foundation and link the asset markets to deep structural parameters in macroeconomics. Further, they provide a unified framework to explore how important the roles that different channels play. However, few works on China's asset market adopt the CCAPM approach.

This paper conducts an empirical exercise to estimate a set of models in the CCAPM framework using China's stock market data and make formal statistical comparisons. These models vary in whether they consider habit formation and/or housing service consumption. Specifically, they consist of the classical CCAPM as a benchmark, a model with both nonhousing consumption and housing service consumption, a habit formation model, and a model with both of the previous two features.

We first estimate the structural parameters in each model by GMM using quarterly data from the launch year of China's stock market, 1991, to 2009. We obtain two main findings. First, habit formation affects stock returns only if housing service consumption is considered. Second, every model considered has some explanatory power and is consistent with the data to certain extent. Such indeterminacy implies that just fixing one model and estimate it may lead to statistically coherent results but conceal the true key mechanism.

Hence, built on the estimates, we conduct cross-model comparisons by examining two measures. The first measure is called the Hansen-Jagannathan Distance. It is a statistical measure, that is, a version of the goodness-to-fit for these nonlinear models estimated by GMM. This measure illustrates how good each model fits the data. The second measure is more economically meaningful. We estimate the model-implied risk premia and examine how large they are compared to the true risk premium across models. Since risk premium is one of the most important research questions that CCAPM tries to answer, the best model should have the largest explanatory power on it.

By these comparisons, we find that the models considering habit formation perform substantially better, and the model that further considers housing service consumption has the best explanatory power. The results imply that one key determinant of China's stock prices is agents' consumption habit, and the effect of the latter is mainly through the channel of housing service consumption.

This paper is built on the development in the CCAPM theory. Incorporating habit formation into CCAPM is among the first development of the classical CCAPM. Examples include Abel (1990), Constantinides (1990), Detemple and Zapatero (1991), and Campbell and Cochrane (1999). They find that risk premium can be moderately better explained with this structure. As for including richer components in consumption, Eichenbaum and Hansen (1990) introduce durable goods consumption to utility function and analyze substitution between durable and non-durable goods. Piazzesi, Schneider and Tuzel (2007) in particular consider housing service consumption. The paper that is closest to our work is Kwan, Leung and Dong (2015). They compare the empirical performance of the models considered in the paper and some other models by applying them to Hong Kong asset market data. Since Hong Kong is a geographically small city and has different institutional and economic backgrounds than Mainland China, their results may be not directly applicable to the scenario considered in this paper. Meanwhile, they do not compare the estimated risk premia across the models.

The rest of the paper is organized as follows. In Section 2 we formally introduce the four 
models. Section 3 introduces the data we use. Section 4 presents the model-wise structural parameter estimates. Section 5 compares the goodness-to-fit and the explanatory power on risk premia across the models. Section 6 concludes.

\section{The Models}

We empirically test the following four models in this paper: classical CCAPM, CCAPM with housing (HCCAPM), CCAPM with habit formation (Habit-CCAPM) and HCCAPM with habit formation (Habit-HCCAPM).

These models differ in how utility is specified, yet they still share the same foundation; a forward-looking representative agent optimizes her life-time utility through asset allocation. Following the standard argument, an implication is that the expected discounted return of the asset, in this paper the stock, is equal to unity. Let $R_{t}$ denote the gross rate of stock return in period $t$, and $M_{t}$ be the stochastic discount factor (SDF, also known as the pricing kernel) in period $t$. We have the following Euler equation

$$
E_{t}\left(M_{t+1} R_{t+1}\right)=1
$$

Equation (1) provides the empirical foundation to estimate and compare different versions of CCAPM. Yet in order to estimate $M_{t+1}$, we need to derive its parametric form first. The form of it varies with how the utility function is formulated. In the rest of this section, we follow Kwan, Leung and Dong (2015) closely and present the formulas of $M_{t+1}$ in each model. We include the key steps in deriving the SDF in each model for completeness.

\subsection{CCAPM}

We first derive the SDF in the classical CCAPM as a benchmark. Suppose the representative agent minimizes her lifetime utility by choosing a single consumption goods $\left\{C_{t}\right\}_{t}$. The value function is as follows.

$$
E_{t} \sum_{j=0}^{\infty} \beta^{j} u\left(C_{t+j}\right) \equiv V_{t}=u\left(C_{t}\right)+\beta E_{t} V_{t+1}
$$

where $\beta \in(0,1)$ is the discount factor. Set up the Bellman equation

$$
\begin{gathered}
V\left(x_{t}, z_{t}\right)=\max _{C_{t}}\left\{u\left(C_{t}\right)+\beta E_{t} V\left(x_{t+1}, z_{t+1}\right)\right\} \\
\text { s.t. } \quad x_{t+1}=R_{t+1}\left(x_{t}-C_{t}\right)
\end{gathered}
$$

where $x_{t}$ is the amount of wealth in period $t$. To obtain an analytical solution for $M_{t+1}$, we assume that the utility function has the form of constant relative risk aversion (CRRA):

$$
u(c)=\frac{c^{1-\rho}-1}{1-\rho}
$$

where $\rho$ both represents the coefficient of relative risk aversion and the inverse elasticity of intertemporal substitution (IES).

Substitute the utility function into the maximization problem and take first order derivative with respect to $C_{t}$ : 


$$
u\left(C_{t}\right)^{\prime}=\beta E_{t} V_{1 t+1} R_{t+1}=C_{t}^{-\rho}
$$

Meanwhile, by the envelop theorem, the derivative with respect to $x_{t}$ is as follows:

$$
V_{1 t}=\beta E_{t} V_{1 t+1} R_{t+1}
$$

By some algebraic manipulation, equations (1), (4) and (5) imply that the SDF in this model is

$$
M_{t+1}=\beta \frac{u\left(C_{t+1}\right)^{\prime}}{u\left(C_{t}\right)^{\prime}}=\beta\left(\frac{C_{t+1}}{C_{t}}\right)^{-\rho}
$$

\subsection{HCCAPM}

In this model, we decomposite the total consumption $C_{t}$ into two categories, non-housing consumption $c_{t}$ and housing service consumption $s_{t}$. Specifically, $C_{t}$ is assumed to have the following CES form:

$$
C_{t}=g\left(c_{t}, s_{t}\right)=\left(c_{t}^{1-\phi}+\omega s_{t}^{1-\phi}\right)^{\frac{1}{1-\phi}}, \quad \omega>0, \phi \geq 0
$$

where $\phi>0$ is the inverse of the elasticity of substitution between housing service consumption and non-housing consumption. The rest of the model is similar to the classical CCAPM.

Set up the Bellman Equation:

$$
\begin{gathered}
V\left(x_{t}, z_{t}\right)=\max _{c_{t}, s_{t}}\left\{u\left(g\left(c_{t}, s_{t}\right)\right)+\beta E_{t} V\left(x_{t+1}, z_{t+1}\right)\right\} \\
\text { s.t. } x_{t+1}=R_{t+1}\left(x_{t}-c_{t}-q_{t} s_{t}\right) \mathrm{s}
\end{gathered}
$$

where $q_{t}$ in the constraint is the relative price of housing service consumption. Define the proportion of the non-housing consumption by $\alpha_{t} \equiv c_{t} / c_{t}+q_{t} s_{t}$. By solving the maximization problem, we obtain

$$
M_{t+1}=\beta\left(\frac{c_{t+1}}{c_{t}}\right)^{-\rho} \cdot\left(\frac{\alpha_{t+1}}{\alpha_{t}}\right)^{\frac{\rho-\phi}{1-\phi}}
$$

\subsection{Habit-CCAPM}

As argued in the literature of habit formation (e.g. Abel, 1990, Constantinides, 1990, Detemple and Zapatero, 1991, and Campbell and Cochrane, 1999), the way that consumption determines an agent's utility in a given period may not just depend on the absolute level of the consumption, but may also depend on its relative level compared to her consumption habit. A straightforward way to implement the idea is to assume it is $c_{t} / X_{t}$ that enters the utility function, where $X_{t} \equiv$ $\left(\bar{c}_{t-1}\right)^{\kappa}$ is the habit level of consumption in period $t$ and $\bar{c}_{t-1}$ is the average level consumption of the entire economy in period $t-1$. When optimizing the objective function, $\bar{c}_{t-1}$ is treated as given. After the first order condition is obtained, we impose an equilibrium condition such that $\bar{c}_{t-1}=c_{t-1}$, i.e., in equilibrium, the representative agent behaves as the average in the economy.

By setting up the Bellman equation with appropriate changes made regarding the utility functions, we have 


$$
M_{t+1}=\beta\left(\frac{c_{t+1}}{c_{t}}\right)^{-\rho}\left(\frac{c_{t}}{c_{t-1}}\right)^{\kappa(\rho-1)}
$$

\subsection{Habit-HCCAPM}

The last model considered in this paper combines the features in the models in Sections 2.3 and 2.4, that is, housing service consumption and habit formation are simultaneously considered.

Let non-housing consumption $c_{t}$ and housing service consumption $s_{t}$ enter the model in the same CES function (7):

$$
C_{t}=\left(c_{t}^{1-\phi}+\omega s_{t}^{1-\phi}\right)^{\frac{1}{1-\phi}}
$$

Then we adjust it by dividing it by the habit level: $X_{t}=\bar{C}_{t-1}^{\kappa}=\left(\bar{c}_{t-1}^{1-\phi}+\omega \bar{s}_{t-1}^{1-\phi}\right)^{\frac{1}{1-\phi}}$ where $\bar{c}_{t-1}$ and $\bar{s}_{t-1}$ are defined in similar ways as in Section 2.3. Solving the Bellman equation that is similar to the previous models, we obtain

$$
M_{t+1}=\beta\left(\frac{c_{t+1}}{c_{t}}\right)^{-\rho}\left(\frac{\alpha_{t+1}}{\alpha_{t}}\right)^{\frac{\rho-\phi}{1-\phi}}\left(\frac{c_{t}}{c_{t-1}}\right)^{\kappa(\rho-1)}\left(\frac{\alpha_{t+1}}{\alpha_{t}}\right)^{\frac{\kappa(1-\rho)}{1-\phi}}
$$

\section{Main Results}

We construct the dataset of China's stock returns and consumption from databases of National Bureau of Statistics of China, People's Bank of China (the central bank of China), China Stock Market \& Accounting Research Database, RESSET and Wind Financial Database. The data cover the period from 1991 to 2009 . The frequency we choose is quarterly because on the one hand, annual data suffer from a small sample size as China stock market was only launched in 1991, while on the other hand, it is well-known that monthly consumption data have larger measurement error.

The construction of the variables appeared in Section 2 is straightforward. The gross stock returns are equal to the net returns from the data plus 1 . We use non-durable goods consumption to proxy non-housing service consumption. The approximation is reasonable because for average households in China, housing service consumption usually takes up the largest share in durable goods consumption. The share $\alpha_{t}$ is thus computed by dividing non-durable goods consumption by the sum of such consumption and housing service consumption.

Note that to guarantee the GMM estimator to yield consistent estimates with valid standard errors, the variables involved in the estimation need to be stationary. Although consumption is often nonstationary, it only enters the SDF in the form of $c_{t+1} / c_{t}$, which is stationary. Similar goes with $\alpha_{t+1} / \alpha_{t}$. Indeed, we perform augmented Dickey-Fuller tests for all the variables in the models. The null hypotheses that each of these series is non-stationary are all rejected at the $1 \%$ level.

Table 1: Summary statistics of the key variables (1990Q1-2019Q4)

\begin{tabular}{|c|c|c|c|c|c|}
\hline & Mean & Std Error & Min & Max & Obs. \\
\hline Stock return $R_{t}$ & 1.0393 & 0.2351 & 0.6235 & 2.2192 & 116 \\
\hline Risk-free asset return $R_{f, t}$ & 1.0086 & 0.0037 & 0.0027 & 0.0160 & 115 \\
\hline Consumption growth $c_{t} / c_{t-1}$ & 1.027 & 0.0909 & 0.8759 & 1.2464 & 119 \\
\hline Non-housing share $\alpha_{t}$ & 0.8643 & 0.0900 & 0.7070 & 0.9955 & 115 \\
\hline
\end{tabular}

Notes: All variables are measured in real terms (baseline: 1990Q1). 
Table 1 shows summary statistics of the key variables. We have two main findings. First, stocks have much larger average net return (3.93\%) and standard error $(0.2351)$ than those of the riskfree asset $(0.86 \%$ and 0.0037 respectively). This observation provides direct evidence for the existence of risk premium in China stock market. Second, on average, non-housing consumption has a large share $(86.43 \%)$ in total consumption in the period the sample covers. This is larger than Hong Kong (77\%, Kwan, Leung and Dong, 2015) and the U.S. (82\%, Piazzesi, Schneider and Tuzel, 2007). Nonetheless, from Figure 1, we see that this share is decreasing over the years, suggesting that the role that housing service consumption plays in determining the asset return may be increasingly important, motivating including housing service consumption in the model.

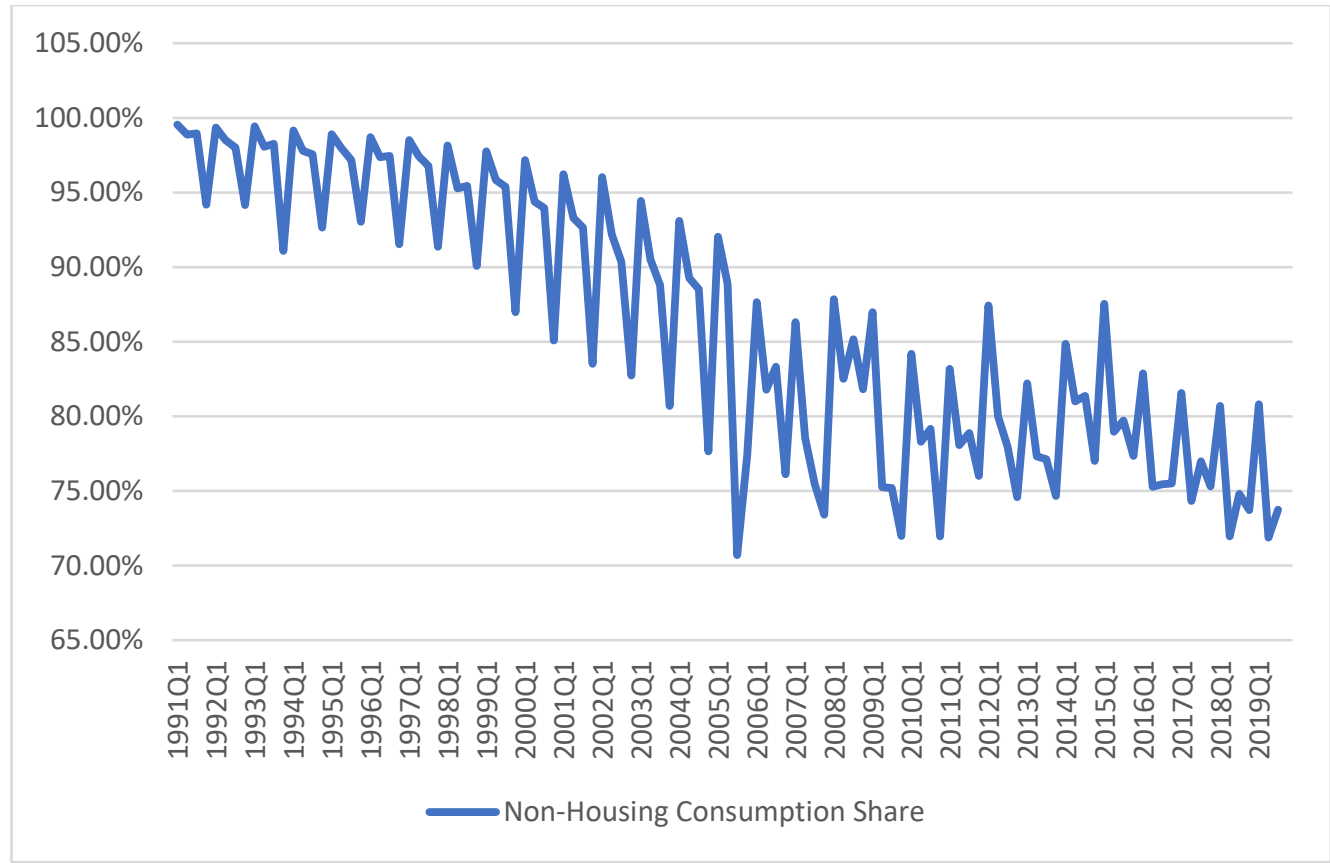

Figure 1: Non-housing consumption share in China from 1991 to 2019

\section{Empirical Results}

Using the dataset constructed in Section 3 and the parametric forms of the SDF yielded in Section 2, we can estimate the structural parameters, and thus the SDF, in each model based on the moment condition (1). In Section 4.1, we first present the estimates of the structural parameters in each model. We find that each model is consistent with the data to certain extent. Meanwhile, habit formation plays a salient role only if housing service consumption is included in the model. In Section 4.2, we compare the empirical performance of each model by the Hansen-Jagannathan distance and by the explanatory power on risk premium. We find that Habit-CCAPM and Habit-HCCAPM perform much better than the other two models, while Habit-HCCAPM has the best overall performance.

\subsection{Structural parameter estimates}

By plugging the formulas of $M_{t+1}$ derived for each model in Section 2 into the Euler equation $E_{t}\left(M_{t+1} R_{t+1}-1\right)=0$, we obtain a moment condition for each model. It is thus natural to adopt GMM to estimate the structural parameters in the models. Note that the moment condition is conditional on the information set at time $t$. Any observable at period $t$ is in this 
information set. In practice, we find a set of observables that have the largest correlation with the moment function $\left(M_{t+1} R_{t+1}-1\right)$ and multiply the moment function by them. In this way, the moment condition is transformed to a set of unconditional moments. These observables selected are called the instrumental variables (IV).

Table 2: Interpretations of the structural parameters

\begin{tabular}{|l|l|}
\hline $\boldsymbol{\beta}$ & Discount factor \\
\hline $\boldsymbol{\rho}$ & Relative risk aversion and inverse of the IES \\
\hline $\boldsymbol{\phi}$ & Inverse of elasticity of substitution between housing and non-housing consumption \\
\hline $\boldsymbol{\kappa}$ & Habit factor \\
\hline
\end{tabular}

Before we present the estimates, it is useful to first discuss the meaning of each structural parameter in the models.

As the discount factor, $\beta$ is smaller than 1 , and is usually deemed no less than 0.9 . A larger $\beta$ implies that the agent is more patient as she cares about utility realized in the future by more. As a consequence of the CRRA utility function, $\rho$ in these models plays two roles. On the one hand, it captures relative risk aversion. The larger $\rho$ is, the more risk averse the agent would be, implying a higher risk premium. In the meantime, $\rho$ is also the inverse of the IES. A larger $\rho$ thus implies the agent is less elastic in substituting consumption of this period by that of the next period. In this respect, a small $\rho$ explains the low risk-free rate. The parameter $\phi$ only shows up in HCCAPM and Habit-HCAPM. As the inverse of the elasticity of substitution between housing service consumption and non-housing consumption, an agent with a larger $\phi$ is less flexible to substitute housing using non-housing consumption. Finally, $\kappa$ captures how large consumption habit formed in the past affects consumption today. As $\bar{c}_{t-1}>1$, the bigger $\kappa$ is, the larger the effect of the past consumption is. When $\kappa=0$, Habit-CCAPM and HabitHCCAPM reduce to CCAPM and HCCAPM, respectively.

Table 3: GMM estimates of the structural parameters

\begin{tabular}{|c|c|c|c|c|}
\hline & CCAPM & HCCAPM & Habit-CCAPM & Habit-HCCAPM \\
\hline & $\mathbf{( 1 )}$ & $\mathbf{( 2 )}$ & $\mathbf{( 3 )}$ & $\mathbf{( 4 )}$ \\
\hline$\hat{\beta}$ & $0.9639^{* * *}$ & $0.9615^{* * *}$ & $0.9620^{* * *}$ & $0.9823^{* * *}$ \\
\hline$\hat{\rho}$ & $(0.0256)$ & $(0.0200)$ & $(0.0231)$ & $(0.0199)$ \\
\hline & 0.7768 & 0.0843 & -0.4452 & $-0.5502^{* *}$ \\
\hline$\hat{\phi}$ & $(1.1796)$ & $(0.3306)$ & $(0.3886)$ & $(0.2753)$ \\
\hline$\hat{\kappa}$ & & 0.3066 & & 5.3964 \\
\hline & & $(0.4288)$ & & $(5.8297)$ \\
\hline J-statistic & 2.9912 & 2.7685 & 2.8273 & $0.6495^{* *}$ \\
\hline$[p-v a l u e]$ & 0.2241 & 0.7356 & 0.5871 & $(0.2743)$ \\
\hline Sample size & 112 & 112 & 113 & 0.4533 \\
\hline
\end{tabular}


Notes: (1) Standard errors are in the parentheses. (2) *, **, *** represent significant at the $10 \%, 5 \%$ and $1 \%$ levels respectively. (3) The instruments used includes lags of $c_{t+1} / c_{t}$, of $R_{t+1}$, and their interactions. All models are overidentified by including more instruments than the structural parameters.

From Table 3, we have the following four findings. First, $\hat{\beta}$ across the models are robust and lying in the expected interval $(0.9,1)$.

Second, $\hat{\rho}$ is small and in most of the models statistically indistinguishable from 0 , explaining the low risk-free rate well. As mentioned earlier, $\rho$ plays two roles. High risk premium is in favor of a large $\rho$ (high relative risk aversion) while low risk-free rate of return needs a small $\rho$ (high IES). From the results, these models tend to explain the low-risk free rate more. Note that in the third and the fourth models, the estimated $\rho$ are negative. Negative intertemporal elasticity of substitution is also documented in Hall (1988). This again suggests the estimates of $\rho$ are more driven by the role of the IES.

Third, housing service consumption is more relevant to habit formation than non-housing consumption is. When not considering housing service consumption, $\hat{\kappa}$ in Column (3) is insignificant. As noted before, when $\kappa=0$, the habit formation model is identical to CCAPM. Indeed, $\hat{\beta}$ in Columns (1) and (3) are very close, while $\hat{\rho}$ in both cases are insignificant. In contrast, estimates in Column (4) are very different from the other columns with a significantly positive $\hat{\kappa}$. The results in Columns (3) and (4) imply that habit is more important in determining housing service consumption. The implication is reasonable since agents tend to have more sticky consumptive behavior for high-value goods.

Fourth, as the parameters in every model are overidentified, we have the luxury to conduct an over-identification test ( $\mathrm{J}$ test) for the validity of each model. From the results, none of the $\mathrm{J}$ test statistics is significant at any reasonable level. Hence, every model is consistent with the data to some extent and has certain explanatory power.

\subsection{Model comparison}

In this section, we formally compare these models by different criteria to select out the model that has the largest explanatory power for China's stock returns. We use the HansenJagannathan distance as a measure of the goodness-to-fit, and the model implied risk premia as the second criterion. We find that both housing service consumption and habit formation improve the goodness-to-fit while Habit-HCCAPM performs best. In terms of the second criterion, Habit-CCAPM and Habit-HCCAPM outperform their counterparts without habit formation in matching the true risk premium.

\subsubsection{Hansen-Jagannathan distance}

The first criterion we consider is the Hansen-Jagannathan distance (HJ distance) which measures the goodness-to-fit of nonlinear models estimated by GMM.

For linear models, popular ways to measure the goodness-to-fit are $R^{2}$, AIC and BIC. These methods measure the overall distance between the residuals and zero. The closer the residuals are to zero, the better the model fits the data. In contrast, in the models studied in this paper, there is no error term, and when estimating them using GMM, no residuals are obtained. Instead, as estimation is built upon the moment condition $E_{t}\left(M_{t+1} R_{t+1}\right)-1=0$, we can measure how close the estimated moment conditions are to zero.

HJ distance formalizes the idea and is a standard approach for model comparison based on GMM. Let $Z_{t}$ be a vector of the instruments adopted. We then transform the conditional moment equation to the following unconditional expectation, as described in Section 4.1: 


$$
E\left[\left(M_{t+1} R_{t+1}-1\right) Z_{t}\right]=0
$$

Plug in the estimated parameters to $M_{t+1}$ in each model, we obtain the estimated SDF $\widehat{M}_{t+1}$. As we consider stock as the only asset, the HJ distance has the following simple form:

$$
H J=\frac{1}{\sqrt{T}} \frac{\sqrt{\sum_{t}\left(\widehat{M}_{t+1} R_{t+1}-1\right)^{2} Z_{t}^{\prime} Z_{t}}}{\sqrt{\sum_{t} R_{t}^{2}}}
$$

That is, the Euclidean distance between $\left(\widehat{M}_{t+1} R_{t+1}-1\right) Z_{t}$ and 0 , scaled by $1 / \sqrt{T \sum_{t} R_{t}^{2}}$. It is also equal to the objective function that GMM estimator minimizes, scaled by $\left(\sqrt{\sum_{t} R_{t}^{2} / T}\right)^{-1}$

Table 4 presents the estimated HJ distance. From the results, it is clear that introducing either housing service consumption or habit formation into the model leads to better goodness-to-fit, and the model with both elements performs best.

Table 4: HJ distance

\begin{tabular}{|c|c|}
\hline CCAPM & 0.0234 \\
\hline HCCAPM & 0.0215 \\
\hline Habit-CCAPM & 0.0199 \\
\hline Habit- HCCAPM & $8.17 \times 10^{-9}$ \\
\hline
\end{tabular}

The results in Table 4 imply that in China's stock market, consumption habit and housing service consumption both play important roles in understanding risk premium and asset returns. To match the consumption habit, higher consumption is needed, and thus higher asset returns are required to finance the consumption. Meanwhile, the effect of consumption habit is mostly through the channel of housing service consumption, echoing the findings in Section 4.1.

\subsubsection{Estimated risk premium}

The second measure we use to compare the models is how well the models match the average risk premium.

To estimate the average risk premium, first note that for the risk-free asset, denote its return by $R_{f, t}$. Following the standard argument, $R_{f, t}$ is treated as deterministic. Then we have

$$
E\left(M_{t+1} R_{f, t+1}\right)=R_{f, t+1} E\left(M_{t+1}\right)
$$

Together with the unconditional Euler equation $E\left(M_{t+1} R_{f t+1}\right)=1$, the following equation holds:

$$
E\left(M_{t+1}\right)=\frac{1}{R_{f, t+1}}
$$

For the stock return, by the definition of covariance, we have

$$
\operatorname{cov}\left(M_{t+1}, R_{t+1}\right)=E\left(M_{t+1}, R_{t+1}\right)-E\left(M_{t+1}\right) E\left(R_{t+1}\right)
$$

Hence, by the unconditional Euler equation for the stock return, 


$$
\begin{gathered}
\operatorname{cov}\left(M_{t+1}, R_{t+1}\right)+E\left(M_{t+1}\right) E\left(R_{t+1}\right)=1 \\
\Rightarrow \operatorname{cov}\left(M_{t+1}, R_{t+1}\right)+\frac{E\left(R_{t+1}\right)}{R_{f, t+1}}=1
\end{gathered}
$$

where the second equation follows from (13). Rearrange the terms, we have

$$
E\left(R_{i, t+1}\right)-R_{f, t+1}=-R_{f, t+1} \cdot \operatorname{cov}\left(M_{t+1}, R_{t+1}\right)
$$

Since $R_{f, t+1}>0$, the SDF is necessarily negatively correlated with the stock return to generate risk premium.

We estimate both sides of equation (14) using data and the estimated SDF. Specifically, we estimate the expectation and the covariance by their sample analogues, and we also average out $R_{f, t+1}$. When computing the sample analogues, we both use the full sample and subsamples of 9 to 10-year span to capture time heterogeneity and check the robustness of the

\begin{tabular}{|c|c|c|}
\hline Model & Estimated Risk Premium & Proportion Explained \\
\hline \multicolumn{3}{|c|}{ A. Full Sample (1991-2019) } \\
\hline Risk Premium & $3.06 \%$ & \\
\hline CCAPM & $-0.14 \%$ & $-4.716 \%$ \\
\hline HCCAPM & $-0.01 \%$ & $-0.454 \%$ \\
\hline Habit-CCAPM & $0.09 \%$ & $3.081 \%$ \\
\hline Habit- HCCAPM & $0.26 \%$ & $8.468 \%$ \\
\hline \multicolumn{3}{|c|}{ B. Year 1991 - 1999} \\
\hline Risk Premium & $6.07 \%$ & \\
\hline CCAPM & $-0.18 \%$ & $-2.910 \%$ \\
\hline HCCAPM & $-0.05 \%$ & $-0.807 \%$ \\
\hline Habit-CCAPM & $0.11 \%$ & $1.811 \%$ \\
\hline Habit- HCCAPM & $0.35 \%$ & $5.799 \%$ \\
\hline \multicolumn{3}{|c|}{ C. Year $2000-2009$} \\
\hline Risk Premium & $3.62 \%$ & \\
\hline CCAPM & $-0.30 \%$ & $-8.285 \%$ \\
\hline HCCAPM & $-0.03 \%$ & $-0.703 \%$ \\
\hline Habit-CCAPM & $0.20 \%$ & $5.543 \%$ \\
\hline Habit- HCCAPM & $0.47 \%$ & $13.074 \%$ \\
\hline \multicolumn{3}{|c|}{ D. Year 2010-2019 } \\
\hline Risk Premium & $-0.20 \%$ & \\
\hline CCAPM & $0.03 \%$ & $-15.358 \%$ \\
\hline HCCAPM & $0.03 \%$ & $-14.959 \%$ \\
\hline Habit-CCAPM & $-0.01 \%$ & $5.535 \%$ \\
\hline Habit- HCCAPM & $-0.01 \%$ & $5.286 \%$ \\
\hline
\end{tabular}
results. The results are presented in Table 5.

Table 5: Risk premia 
the ratio between the estimated risk premium from each model and the one directly computed from data.

Table 5 provides two key findings. First, without considering habit formation, the signs of the estimated risk premia are opposite with the true average risk premia directly computed from the data. When considering habit formation, the signs become coherent with the true premia. From equation (14), the wrong signs imply that the estimated SDF in those models and the stock returns co-move in the same direction, contradicting the theory. One possibility is that these estimates of the SDF series neglect some sticky behavior and thus are overall shifted to the right. That the signs are correctly estimated in Habit-CCAPM and Habit-HCCAPM provides evidence that habit in consumption results in such stickiness.

Second, with habit formation, considering housing service substantially increases the explanatory power on risk premium. Overall, Habit-HCCAPM explains risk premium by 5\% more than Habit-CCAPM. In the period from 1991 to 1999, and from 2000 to 2009, it outperforms the latter by about $4 \%$ and $7.5 \%$, respectively.

To sum up, habit formation and housing service consumption are both important determinants of risk premium in China's stock market.

\section{Conclusion}

This paper estimates and compares four different versions of CCAPM using quarterly China's stock market data from 1991 to 2019. The models vary by whether they consider housing service consumption and/or habit formation.

We find that all these models are consistent with the data to certain extent. Further, the effect of habit formation on stock returns is mainly through the channel of housing service consumption. Across the models, we examine their goodness-to-fit by comparing their HJ distances and evaluate how their explanatory power on risk premium vary. We find that the model with both habit formation and housing service consumption performs best with respect to both criteria. In particular, habit formation is a necessary mechanism to correctly estimate the sign of risk premium, and when housing service consumption is considered as well, the explanatory power substantially increases.

The findings imply that even if a CCAPM model in itself fits the data well to some extent, the fact may not conclusively justify the model should be adopted. The model may still be inferior to other candidates into which richer features are incorporated. In our context, habit formation and housing service consumption are two important elements in understanding China's stock market.

One limitation of the paper is that it only adopts the CRRA-type utility function, so relative risk aversion and the IES are indistinguishable. This may be the reason that even the richest model considered in this paper only has limited explanatory power. In future research, it is interesting to apply other types of preferences to China's stock return data to see if it further improves the results through housing service consumption and habit formation. 


\section{References}

[1] Lucas Jr, R. (1978). Asset prices in an exchange economy. Econometrica, Vol. 46, No. 6, pp. 1429-1445.

[2] Mehra, R., and Prescott, E.C. (1985). The equity premium: A puzzle. Journal of Monetary Economics, 15.2, pp. 145-161.

[3] Campbell, J.Y. (1986). Bond and stock returns in a simple exchange model. The Quarterly Journal of Economics, 101.4, pp. 785-803.

[4] Abel, A.B. (1990). Asset Prices under habit formation and catching up with the Joneses. American Economic Review, Vol. 80, No. 2, pp. 38-42.

[5] Constantinides, G.M. (1990). Habit formation: A resolution of the equity premium puzzle. Journal of Political Economy, 98.3, pp. 519-543.

[6] Detemple, J.B., and Zapatero, F. (1991). Asset prices in an exchange economy with habit formation. Econometrica, Vol. 59, No. 6, pp. 1633-1657.

[7] Campbell, J.Y., and Cochrane, J.H. (1999). By force of habit: A consumption-based explanation of aggregate stock market behavior. Journal of Political Economy, 107.2, pp. 205-251.

[8] Eichenbaum, M. and Hansen, L.P. (1990). Estimating models with intertemporal substitution using aggregate time series data. Journal of Business \& Economic Statistics, 8.1, pp. 53-69.

[9] Piazzesi, M., Schneider, M. and Tuzel, S. (2007). Housing, consumption and asset pricing. Journal of Financial Economics, 83.3, pp. 531-569.

[10]Kwan, Y.K., Leung, C.K.Y., and Dong, J. (2015). Comparing consumption-based asset pricing models: The case of an Asian city. Journal of Housing Economics, 28, pp. 18-41.

[11]Hall, R.E. (1988). Intertemporal substitution in consumption. Journal of Political Economy, 96.2, pp. 339-357. 